\title{
DECOMPOSITIONS OF RIGID SPACES
}

\author{
FONS VAN ENGELEN AND JAN VAN MILI
}

\begin{abstract}
We give an example of a rigid subspace of $\mathbf{R}$ which can be decomposed into two homeomorphic homogeneous parts, and of a rigid subspace of $\mathbf{R}$ which can be decomposed into two homeomorphic rigid parts.
\end{abstract}

1. Introduction. All spaces under discussion are separable metric. In the past few years, a number of papers have been published presenting examples of decompositions of spaces into homeomorphic parts. By Menu [3] and van Mill [5], decompositions of $\mathbf{R}$ into homeomorphic homogeneous parts were constructed. The question of Arhangelskil̆, whether "nice" (homogeneous) spaces could be partitioned into two homeomorphic, very "bad" (rigid) subspaces was answered by van Mill and Wattel in [6], where such partitions were constructed for the circle and many other spaces. A decomposition of $\mathbf{R}$ into two homeomorphic rigid parts has recently been obtained by van Engelen [1].

As becomes clear from the above, up to now attention has been focused upon decompositions of well-known homogeneous spaces. The aim of this note is to consider the reverse situation: we construct a very bad (rigid) space which can be decomposed into two homeomorphic nice (homogeneous) subspaces. An example of a rigid space which can be partitioned into two homeomorphic rigid subspaces is also given.

2. Preliminaries. Let $f: X \rightarrow Y$ be a function; the domain and range of $f$ will be denoted by $\operatorname{dom}(f)$ and $\operatorname{ran}(f)$, respectively. If $f$ is a homeomorphism, we will write $f: X \approx Y$; if $X=Y$, then $f$ is called an autohomeomorphism of $X$. A topological space $X$ is rigid if the identity is its only autohomeomorphism, and homogeneous if for each $x, y \in X$ there exists an autohomeomorphism $f$ of $X$ with $f(x)=y$.

Cardinals are initial ordinals, and an ordinal is the set of its predecessors; the cardinality of a set $A$ is denoted by $|A| ; \boldsymbol{\aleph}_{0}$ is the first infinite ordinal.

If $A$ and $B$ are subsets of $\mathbf{R}$, then $A+B=\{a+b: a \in A, b \in B\}$. If $A=\{x\}$, then we will write $x+A$ instead of $\{x\}+A ; A B, x B$, and $B / x$ are defined similarly.

In the construction of our examples we will use the technique of "killing homeomorphisms", originally due to Kuratowski, and successfully employed by many authors. A fundamental weapon will be the following theorem:

Received by the editors December 28, 1982.

1980 Mathematics Subject Classification. Primary 54G20, 54C99.

Key words and phrases. Rigid, homogeneous. 
2.1. TheOrem (LAVRentiefF [2]). Let $X$ and $Y$ be completely metrizable, and let $A \subset X, B \subset Y$; let $h: A \approx B$. Then there are $G_{\delta}$ 's $A^{\prime}$ in $X$ and $B^{\prime}$ in $Y$ such that $A \subset A^{\prime}, B \subset B^{\prime}$, and $h$ can be extended to a homeomorphism $h^{\prime}: A^{\prime} \approx B^{\prime}$.

We will also need the following result.

2.2. Theorem (van Mill [5]). Let $A \subset \mathbf{R}$ be such that $A+\mathbf{Q}=A$. Then $A$ is homogeneous.

3. The examples. We will now present an example of a dense and rigid subspace of $\mathbf{R}$ which is the disjoint union of two dense homeomorphic, homogeneous subspaces. We start with some notation.

(i) $\mathbf{Q}_{\pi}=\left\{\sum_{i=1}^{k} \pi^{n_{i}} q_{i}: n_{i} \in \mathbf{Z}, q_{i} \in \mathbf{Q}, k \in \mathbf{N}\right\}$; note that $\left|\mathbf{Q}_{\pi}\right|=\boldsymbol{\aleph}_{0}$.

(ii) $A(x)=\cup_{k \in \mathbf{Z}}\left(\pi^{k} x+\mathbf{Q}_{\pi}\right)$; note that $|A(x)|=\boldsymbol{\aleph}_{0}$, and that $y \in A(x)$ iff $x \in A(y)$.

(iii) $\mathscr{F}=\left\{f: \operatorname{dom}(f), \operatorname{ran}(f)\right.$ are $G_{\delta}$ 's in $\mathbf{R}$, and $\left.f: \operatorname{dom}(f) \approx \operatorname{ran}(f)\right\}$;

(iv) $\mathcal{G}=\left\{f \in \mathcal{F}:|\{x \in \operatorname{dom}(f): f(x) \notin A(x)\}|=2^{\aleph_{0}}\right\}$; since $|\mathcal{G}| \leqslant 2^{\boldsymbol{N}_{0}}$, we can enumerate $\mathcal{G}$ as $\left\{f_{\alpha}: \alpha<2^{\aleph_{0}}\right\}$.

Inductively, find $x_{\alpha} \in \operatorname{dom}\left(f_{\alpha}\right)$ for $\alpha<2^{\kappa_{10}}$, such that the following conditions are satisfied:

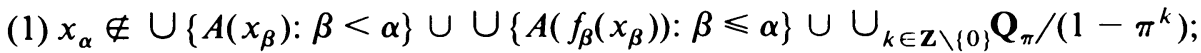

(2) $f_{\alpha}\left(x_{\alpha}\right) \notin \cup\left\{A\left(x_{\beta}\right): \beta \leqslant \alpha\right\}$.

The construction is a triviality; for details, see [1], [4] or [6]. Now put

$$
X_{1}=\bigcup_{\alpha<2^{\aleph_{0}}}\left(x_{\alpha}+\mathbf{Q}\right), \quad X_{2}=\bigcup_{\alpha<2^{\aleph_{1}}} \pi\left(x_{\alpha}+\mathbf{Q}\right) \text {. }
$$

Finally, let $X=X_{1} \cup X_{2}$; we claim that $X$ is as required.

3.1. LEMMA. (a) $X_{1} \approx X_{2}$.

(b) $X_{1}$ and $X_{2}$ are homogeneous.

(c) $X_{1} \cap X_{2}=\varnothing$.

Proof. (a) is trivial, and (b) follows immediately from Theorem 2.2. For (c), suppose that $x_{\alpha}+q=\pi x_{\beta}+\pi q^{\prime}$ for certain $\alpha, \beta<2^{\aleph_{0}}$ and $q, q^{\prime} \in \mathbf{Q}$. Then $x_{\alpha} \in A\left(x_{\beta}\right)$, hence $\alpha=\beta$ because of (1) above. So $x_{\alpha}(1-\pi)=\pi q^{\prime}-q$, whence $x_{\alpha} \in \mathbf{Q}_{\pi} /(1-\pi)$, contradicting (1).

The following lemma is similar to Lemmas 3.2 and 3.3 in [4]; the easy proof is omitted.

3.2. Lemma. (a) $\left\{x_{\alpha}: \alpha<2^{\kappa_{0}}\right\}$ intersects every Cantor set of $\mathbf{R}$.

(b) If $U \subset X$ is open, and $\mathscr{D}$ is a countable family of nowhere dense subsets of $X$, then $|U \backslash \cup \mathscr{D}|=2^{\aleph_{0}}$.

We will now prove the main theorem.

3.3. Theorem. $X$ is rigid. 
Proof. Suppose $f: X \approx X$; we must prove that $f$ is the identity. By Theorem 2.1, there exist $G_{\delta}$ 's $S$ and $T$ in $\mathbf{R}$ such that $f$ can be extended to a homeomorphism $f^{\prime}$ : $S \approx T$. If $\left|\left\{x \in S: f^{\prime}(x) \notin A(x)\right\}\right|=2^{\aleph_{0}}$, then $f^{\prime} \in \mathcal{G}$, say $f^{\prime}=f_{\alpha}$; then $x_{\alpha} \in X$, hence $f_{\alpha}\left(x_{\alpha}\right)=f\left(x_{\alpha}\right) \in X$. So $f_{\alpha}\left(x_{\alpha}\right) \in\left\{x_{\beta}+q, \pi\left(x_{\beta}+q\right)\right\}$ for some $\beta<2^{\kappa_{0}}, q \in$ Q. But then $f_{\alpha}\left(x_{\alpha}\right) \in A\left(x_{\beta}\right)$, contradicting (1) or (2). We conclude that $\mid\{x \in S$ : $\left.f^{\prime}(x) \notin A(x)\right\} \mid<2^{\aleph_{0}}$, and thus also that $|\{x \in X: f(x) \notin A(x)\}|<2^{\aleph_{0}}$.

For $y \in \mathbf{Q}_{\pi}, k \in \mathbf{Z}$ put $X_{k, y}=\left\{x \in X: f(x)=\pi^{k} x+y\right\}$. Then $X_{k, y}$ is closed in $X$. Assume that for a certain $k \in \mathbf{Z}, y \in \mathbf{Q}_{\pi}$ we have that $X_{k, y}$ has nonempty interior (in $X$ ). By Lemma 3.2(a), $x_{\alpha} \in \operatorname{Int} X_{k, y}$ for some $\alpha<2^{\aleph_{0}}$; then $f\left(x_{\alpha}\right)=\pi^{k} x_{\alpha}+y$. Also, $f\left(x_{\alpha}\right) \in X$.

Case 1. $f\left(x_{\alpha}\right)=\pi x_{\beta}+\pi q$ for some $\beta<2^{\aleph_{0}}, q \in \mathbf{Q}$. Then $\pi x_{\beta}+\pi q=\pi^{k} x_{\alpha}+y$, or, equivalently, $x_{\beta}=\pi^{k-1} x_{\alpha}+y / \pi-q \in \pi^{k-1} x_{\alpha}+\mathbf{Q}_{\pi} \subset A\left(x_{\alpha}\right)$. Hence $\alpha=\beta$ and therefore $x_{\alpha}\left(1-\pi^{k-1}\right) \in \mathbf{Q}_{\pi}$. From (1) it follows that $k=1$, and hence $y=\pi q$. So for all $x \in X_{k, y}$ we have that $f(x)=\pi x+\pi q$. Again by Lemma 3.2(a), $\pi x_{\delta} \in$ Int $X_{k, y}$ for some $\delta<2^{\boldsymbol{N}_{0}}$. Hence $f\left(\pi x_{\delta}\right)=\pi^{2} x_{\delta}+\pi q$, and also $f\left(\pi x_{\delta}\right) \in X$.

Case (i). $f\left(\pi x_{\delta}\right)=x_{\gamma}+q^{\prime}$ for some $\gamma<2^{\aleph_{0}}, q^{\prime} \in \mathbf{Q}$. Then $x_{\gamma}=\pi^{2} x_{\delta}+\pi q-q^{\prime}$ $\in \pi^{2} x_{\delta}+\mathbf{Q}_{\pi} \subset A\left(x_{\delta}\right)$, so $\gamma=\delta$ and $x_{\delta}\left(1-\pi^{2}\right) \in \mathbf{Q}_{\pi}$, contradicting (1).

Case (ii). $f\left(\pi x_{\delta}\right)=\pi x_{\gamma}+\pi q^{\prime}$ for some $\gamma<2^{\kappa_{0}}, q^{\prime} \in \mathbf{Q}$. As above, $\gamma=\delta$ and $x_{\delta}(1-\pi) \in \mathbf{Q}_{\pi}$, again contradicting (1).

Case 2. $f\left(x_{\alpha}\right)=x_{\beta}+q$ for some $\beta<2^{\kappa_{0}}, q \in \mathbf{Q}$. As in Case $1, \beta=\alpha$, and $x_{\alpha}\left(1-\pi^{k}\right) \in \mathbf{Q}_{\pi}$; hence $k=0$ and $y=q$, so for all $x \in X_{k, y}$ we have that $f(x)=x$ $+q$. If $\delta<2^{\aleph_{0}}$ is such that $\pi x_{\delta} \in$ Int $X_{k, y}$, then $f\left(\pi x_{\delta}\right)=\pi x_{\delta}+q \in X$.

Case (i). $f\left(\pi x_{\delta}\right)=x_{\gamma}+q^{\prime}$ for some $\gamma<2^{\aleph_{0}}, q^{\prime} \in \mathbf{Q}$. Then $\gamma=\delta$ and $x_{\delta}(1-\pi)$ $\in \mathbf{Q}_{\pi}$, a contradiction.

Case (ii). $f\left(\pi x_{\delta}\right)=\pi x_{\gamma}+\pi q^{\prime}$ for some $\gamma<2^{\aleph_{0}}, q^{\prime} \in \mathbf{Q}$. Then $\gamma=\delta$ and $\pi q^{\prime}=q$, whence $q=0$.

From the above it follows that Int $X_{k, y}=\varnothing$ if $k \neq 0$ or $q \neq 0$. So by Lemma 3.2(b), for every open $U \subset X,\left|U \backslash \cup\left\{X_{k, y}: k \in \mathbf{Z}, y \in \mathbf{Q}_{\pi},(k, y) \neq(0,0)\right\}\right|=2^{\aleph_{0}}$, whereas $\left|U \backslash \cup\left\{X_{k, y}: k \in \mathbf{Z}, y \in \mathbf{Q}_{\pi}\right\}\right|<2^{\boldsymbol{\aleph}_{0}}$. Hence $X_{0,0}$ is dense in $X$ and therefore equal to $X$; so $f$ must be the identity.

The construction of our second example is very similar to that of the first one; hence we will only give a sketch of the proof of the following theorem:

3.4. TheOREM. There exists a rigid subspace $Y$ of $\mathbf{R}$ such that $Y=Y_{1} \cup Y_{2}$, where $Y_{1}, Y_{2}$ are dense, rigid, disjoint and homeomorphic subspaces of $Y$.

Proof. Put $A(x)=x+\mathbf{Z}$, and replace (1) in the construction of the first example by: (1)' $x_{\alpha} \notin \cup\left\{A\left(x_{\beta}\right): \beta<\alpha\right\} \cup \cup\left\{A\left(f_{\beta}\left(x_{\beta}\right)\right): \beta \leqslant \alpha\right\}$. Define $Y_{1}=\left\{x_{\alpha}: \alpha<\right.$ $\left.2^{\aleph_{0}}\right\}$ and $Y_{2}=Y_{1}+1$. Then $Y=Y_{1} \cup Y_{2}$ is as required.

4. Remark. Considering the examples in this note, and in previous papers, one might wonder whether there exist spaces which cannot be decomposed into two homeomorphic rigid or homogeneous parts; or, even better, a space which cannot be decomposed into two homeomorphic parts at all. The easiest, and most insipid example is the one point space. A somewhat less uninteresting example is a 
convergent sequence (with its limit), but of course one would like to have a space without isolated points. Such a space can be obtained by replacing the $n$th point of the sequence by the $n$-dimensional cube (use a dimension argument). However, all these examples behave the way they do because there is an odd number of bad points (viz one). It would be nice to have an example of a homogeneous space (infinite) which cannot be partitioned into two homeomorphic parts.

\section{RETERENCES}

1. F. van Engelen, $A$ decomposition of $\mathbf{R}$ into two homeomorphic rigid parts, Topolgy Appl. (to appear).

2. M. Lavrentieff, Contribution à la théorie des ensembles homéomorphes, Fund. Math. 6 (1924), $149-160$.

3. J. Menu, $\boldsymbol{A}$ partition of $\mathbf{R}$ in two homogeneous and homeomorphic parts (to appear).

4. J. van Mill, Strong local homogeneity does not imply countable dense homogeneity, Proc. Amer. Math. Soc. 84 (1982), 143-148.

5. __ Homogeneous subsets of the real line, Compositio Math. 46 (1982), 3-13.

6. J. van Mill and E. Wattel, Partitioning spaces in homeomorphic rigid parts, Colloq. Math. (to appear).

MAThematisch INSTITUUt, Universiteit VAN AmSterdam, RoetersStraAt 15, 1018 WB AmSTERDAM, THE NETHERLANDS

Subfaculteit Wiskunde, Vrije Universiteit, De Boelelaan 1081, 1081 HV Amsterdam, The NETHERLANDS 